\title{
Lack of maturation with anti-leptin receptor antibody in melanoma but not in nevi
}

\author{
A Hafeez Diwan ${ }^{1}$, Shyam M Dang ${ }^{2}$, Victor G Prieto ${ }^{1}$ and Julie A Ellerhorst ${ }^{2}$ \\ ${ }^{1}$ Department of Pathology, University of Texas MD Anderson Cancer Center, Houston, TX, USA and \\ ${ }^{2}$ Department of Experimental Therapeutics, University of Texas MD Anderson Cancer Center, Houston, \\ TX, USA
}

\begin{abstract}
We have previously shown thryotropin-releasing hormone expression in nevi and melanoma. Thryotropinreleasing hormone regulation by leptin has been shown in the hypothalamus. The present study was therefore undertaken to evaluate leptin and leptin receptor in nevi and melanoma. Leptin receptor expression as assessed using an anti-leptin receptor antibody showed uniform expression throughout the lesion in 14 of 17 melanomas; 3 melanomas lacked leptin receptor immunoreactivity. In contrast, out of 15 nevi, 10 showed weak to moderate leptin receptor immunoreactivity, with positivity present only in the superficial dermal component. Thus, maturation was present in nevi but not in melanoma with the anti-leptin receptor antibody $(P<0.0001)$. Anti-leptin antibody, in contrast, did not show a significant difference in maturation between nevi and melanoma. We also compared leptin receptor in Spitz nevi and melanoma, as the two can sometimes be difficult to distinguish. Spitz nevi showed moderate to strong immunopositivity. Of 19 Spitz nevi, 7 showed lack of maturation, a finding statistically significant from both melanoma and nevi. Our results suggest a role for leptin receptor in melanoma, and we show for the first time that melanomas show more intense immunoreactivity as compared to nevi (but not Spitz nevi) and that maturation with anti-leptin receptor antibody may be a diagnostically useful tool in distinguishing melanomas, especially nevoid ones, from nevi in difficult cases. Modern Pathology (2009) 22, 103-106; doi:10.1038/modpathol.2008.166; published online 3 October 2008
\end{abstract}

Keywords: lack of maturation; melanoma; nevi; leptin receptor

Thryotropinreleasing hormone has been described in melanoma and nevi. Thryotropin-releasing hormone has been shown to stimulate proliferation of melanoma cell lines. Moreover, in patients with melanoma, dypslastic nevi exhibit increased thryotropin-releasing hormone compared with such nevi in individuals without melanoma. It has been speculated that a thryotropin-releasing hormone may act as a melanoma autocrine growth factor. ${ }^{1}$

In the hypothalamus, thryotropin-releasing hormone is produced in response to hypothyroidism. Another stimulus for thryotropin-releasing hormone production is leptin. Fasting, resulting in low leptin levels, reduces thryotropin-releasing hormone biosynthesis. Conversely, leptin has been shown to both directly and indirectly stimulate thryotropin-releasing hormone production. ${ }^{2}$ A leptin receptor has a wide anatomic distribution

Correspondence: Dr AH Diwan, MBBS, PhD, Department of Pathology, University of Texas MD Anderson Cancer Center, 1515 Holcombe Blvd, Unit 85, Houston, TX 77030, USA.

E-mail: ahdiwan@mdanderson.org

Received 7 April 2008; revised and accepted 24 June 2008; published online 3 October 2008 in the mouse (including in the spleen, adrenal, liver, testes, and lung). ${ }^{3}$ Pertinently, in addition to its metabolic role leptin has numerous other effects, including mitogenesis in several tumor and non-tumor cell lines. ${ }^{4}$

Given these findings, we sought to determine the expression of leptin and its receptor in melanoma and nevi. We show that leptin receptor expression in nevi is weaker and shows maturation (ie loss of expression with descent in the dermis) in contrast to melanomas and Spitz nevi. This may be a useful diagnostic tool in distinguishing melanomas (especially nevoid ones) from nevi in challenging cases.

\section{Materials and methods}

Using an IRB-approved protocol, paraffin-embedded archived tissue of melanoma (17 cases), nevi (15 cases), and Spitz nevi (19 cases) was obtained. Immunohistochemical analysis of melanoma and nevi sections for leptin and leptin receptor expression was performed using commercially available polyclonal antibodies (R\&D Systems, Minneapolis, MN, USA). Briefly, tissue sections were deparaffinized in xylenes, and rehydrated in graded 
concentrations (100-85\%) of ethanol. Sections were placed in Antigen Unmasking Solution (Vector Laboratories, Burlingame, CA, USA) and microwaved intermittently for $10 \mathrm{~min}$, to maintain boiling temperature. After cooling, the slides were placed in $3 \% \mathrm{H}_{2} \mathrm{O}_{2}$ in cold methanol for $15 \mathrm{~min}$ to block endogenous peroxidase activity. This step was followed by permeabilization with $0.05 \%$ Triton X-100 (Sigma) in phosphate-buffered saline for $15 \mathrm{~min}$. An avidin-biotin-peroxidase complex (ABC) kit (Vectastain; Vector Laboratories) was then used for antigen detection. After a 30-min incubation with blocking serum, the primary antibody was applied and incubated for $2 \mathrm{~h}$ at room temperature. The slides were then washed, incubated for $30 \mathrm{~min}$ with secondary biotinylated antibody, followed by a 30-min incubation with the ABC reagent. The immunolabeling was developed with the chromogen 3-amino-9-ethylcarbazole. Hematoxylin was applied as a counterstain. For Spitz nevi, only leptin receptor antibody was tested (as additional sections were not available for study with leptin antibody).

The results were recorded with respect to intensity of staining ( 0 , negative; 1 , weak; 2 , moderate; and 3 , strong) and percentage $(0,<5 \% ; 1,5-25 \% ; 2$, $26-75 \%$; and $3,>75 \%$ ). Fisher's exact test was used to determine statistical significance.

\section{Results}

\section{Leptin-Receptor in Melanoma and Nevi}

The majority of melanoma (14 of 17) showed at least moderate intensity throughout the lesion (Figures 1 and 2). Three melanomas lacked leptin receptor reactivity. There was no maturation evident, ie, both the upper and lower portions of melanoma showed similar immunopositivity.

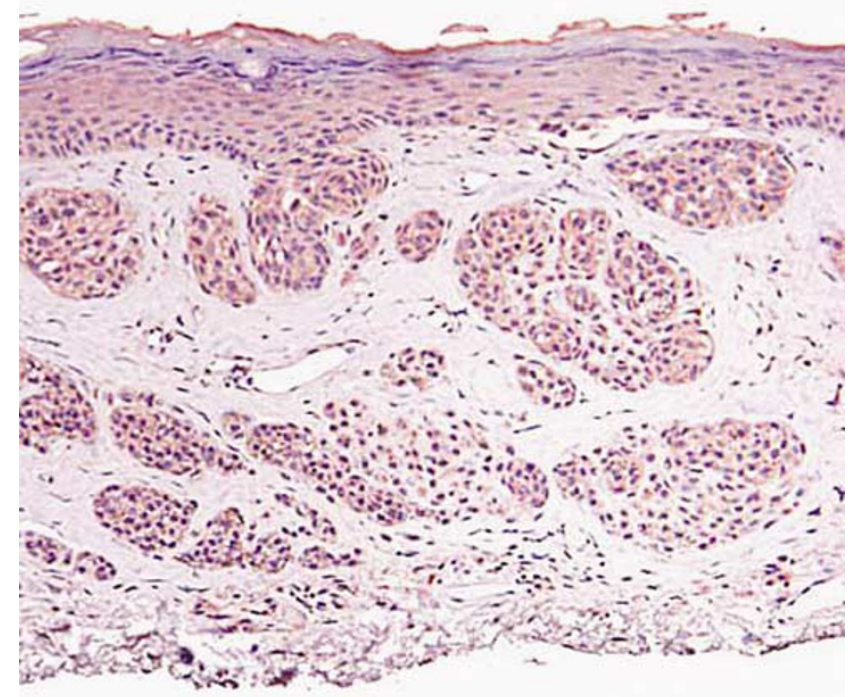

Figure 1 Melanoma shows lack of maturation with anti-LR antibody (original magnification $\times 40$ ).
In contrast, of 15 nevi evaluated, leptin receptor immunoreactivity was weak in 10 of 15 cases. In all the leptin receptor immunoreactive nevi, however, maturation was evident, with weak positivity in the
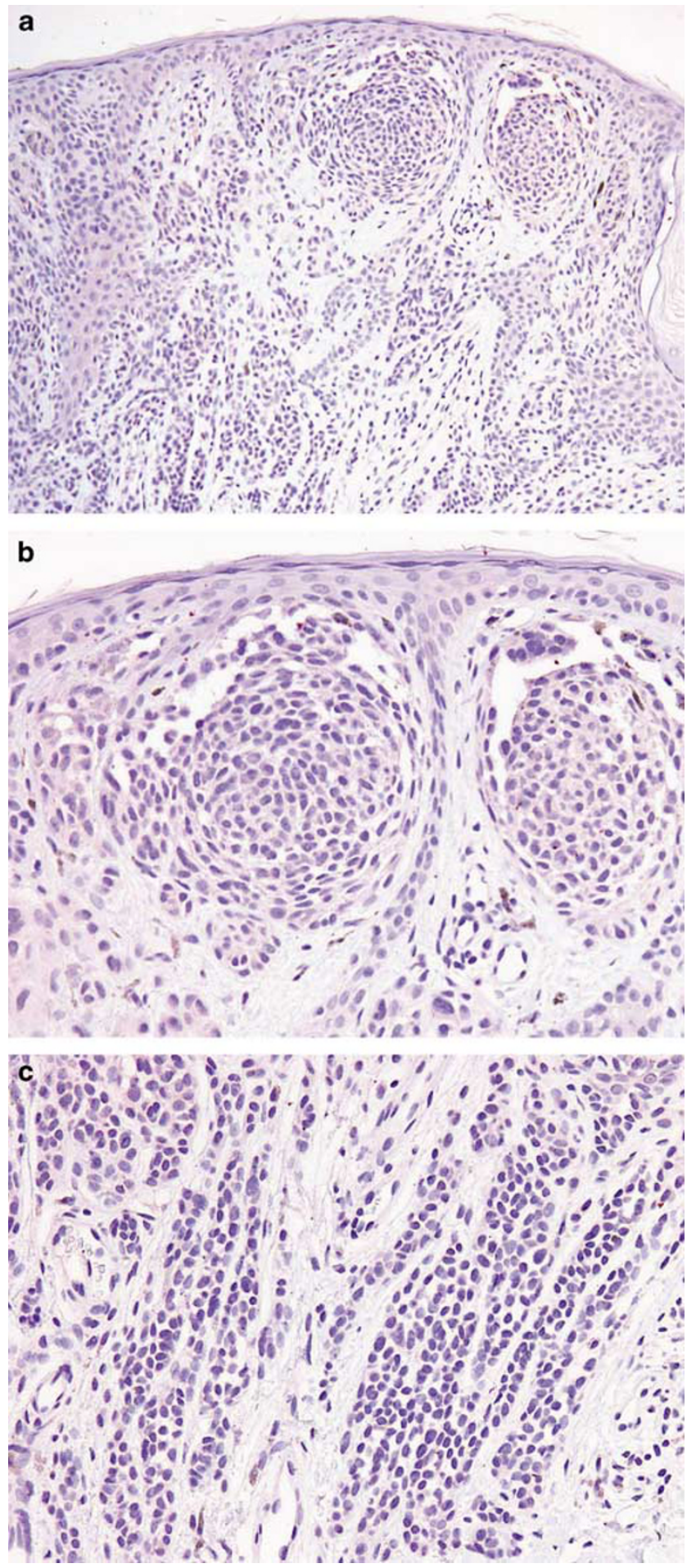

Figure 2 Nevus shows faint immunoreactivity with anti-LR with maturation (a: original magnification $\times 40$; b: top of nevus, original magnification $\times 100$; $\mathbf{c}$ : lower portion of nevus, original magnification $\times 100)$. 
upper portion of the lesions. If only immunoreactive lesions were selected, the proportions of lack of maturation were 14 of 14 melanomas and 0 of 10 nevi. This finding was statistically significant $(P<0.0001)$.

Conversely, maturation was present in all 10 nevi with any immunoreactivity, and none of the immunoreactive melanomas.

\section{Leptin in Melanoma and Nevi}

All the melanoma specimens evaluated showed leptin immunoreactivity (13 of 13, 4 cases could not be evaluated). Maturation was generally absent. However, of the 15 nevi, 13 were leptin positive and 8 lacked maturation. The difference in maturation or lack of it between melanoma and nevi was not statistically significant.

\section{Leptin Receptor in Spitz Nevi}

Unlike nevi, Spitz nevi showed generally moderate to strong leptin receptor immunoreactivity (in 12 of 19) with a few (3 of 19) exhibiting weaker immunoreactivity. The rest (4 of 19) were leptin receptor negative. Loss of maturation was seen in 7 of 19 cases (in 4 cases, no dermal component was present and so maturation could not be evaluated in those cases). Only 2 of 19 cases showed maturation. When lack of maturation was considered in only the immunoreactive lesions, the proportions showing this finding were 7 of 15 Spitz nevi as compared to all 14 melanomas $(P=0.0022)$ and none of the nevi $(P=0.02)$. Conversely, maturation was present in 2 of 15 Spitz nevi as compared to 0 of 14 melanomas (a nonsignificant finding) and 10 of 10 nevi $(P<0.0001)$.

\section{Discussion}

The most significant finding described herein is the difference in leptin receptor expression between nevi and melanoma/Spitz nevi with respect to maturation. Nevi showed generally much weaker leptin receptor immunoreactivity than either melanoma or Spitz nevi, and maturation was present in all the nevi evaluated. Conversely, maturation was absent in all the melanomas that had leptin receptor immunoreactivity. The findings are similar to those seen with the marker HMB-45. In the diagnostic arena, this finding may be of great use in the evaluation of nevoid melanomas that can be very hard to diagnose correctly at times. The finding of lack of maturation with the leptin receptor antibody may lend further support to a diagnosis of nevoid melanoma, in addition to histologic findings and evaluation with the HMB-45 antibody.

Spitz nevi can also pose diagnostic difficulties as at times it may not be possible to identify a lesion as either a Spitz nevus or a melanoma. However, our findings suggest that leptin receptor expression cannot be used reliably to tell the two entities apart.
Both Spitz nevi and melanoma exhibited moderate to strong leptin receptor immunopositivity. But, as two of the Spitz nevi studied did exhibit maturation, a spitzoid melanocytic lesion with maturation might be less likely to be a melanoma, with the caveat that this finding was not statistically significant.

Overall, the presence of maturation with antileptin receptor antibody, as it was not seen in any of melanomas evaluated, would be a very helpful adjunct in the diagnosis of melanoma.

With respect to leptin itself, there was no significant difference between nevi and melanomas. It is interesting to speculate that nevi may not be subject to regulation by leptin, in contrast to melanomas/Spitz nevi. This finding needs further study to be fully evaluated.

The presence of leptin receptor in melanomas and Spitz nevi is intriguing at a biological level. One possibility may be that in situations where leptin is elevated (eg in obesity), the presence of leptin receptors in melanomas (and Spitz nevi) may allow these to be regulated by leptin, perhaps via leptin acting on its receptor and stimulating thryotropinreleasing hormone, which in turn would cause proliferation of the melanoma. As noted in the introduction, leptin has been shown to stimulate thryotropin-releasing hormone production in the hypothalamus, and thryotropin-releasing hormone has been shown to stimulate proliferation in melanoma cell lines. But this can only be speculated at this time and needs further study. We are currently engaged in evaluating the role of leptin in melanoma cell lines.

That there may be a connection between leptin and melanoma has been addressed in a few studies. Gogas et $a l^{5}$ have found that melanoma risk was associated with serum leptin levels in a retrospective study. In a cDNA microarray study, Torisu-Itakura et $a l^{6}$ have shown that among others leptin genes were overexpressed in sentinel lymph nodes with melanoma as compared to non-melanoma sentinel nodes. However, one study casts doubt about leptin involvement in melanoma: As leptin levels are increased in obesity, Brandon et al $P^{7}$ have evaluated knockout mice and shown that obesity, in their system, enhanced melanoma growth independent of leptin levels. But additional study is needed to adequately characterize the role of leptin in melanoma.

Overall, the findings presented highlight for the first time the usefulness of the intensity and pattern of leptin receptor expression, similar to the pattern seen with HMB-45, in the diagnosis of melanoma. As with other markers, however, the finding has to be interpreted in the context of the complete histologic and clinical picture, to avoid misdiagnosis.

\section{References}

1 Ellerhorst JA, Naderi AA, Johnson MK, et al. Expression of thyrotropin-releasing hormone by human melanoma and nevi. Clin Cancer Res 2004;10:5531-5536. 
2 Nillni EA, Vaslet C, Harris M, et al. Leptin regulates prothyrotropin-releasing hormone biosynthesis. Evidence for direct and indirect pathways. J Biol Chem 2000;275:36124-36133.

3 Hoggard N, Mercer JG, Rayner DV, et al. Localization of leptin receptor mRNA splice variants in murine peripheral tissues by RT-PCR and in situ hybridization. Biochem Biophys Res Commun 1997;232:383-387.

4 Gat-Yablonski G, Phillip M. Leptin and regulation of linear growth. Curr Opin Clin Nutr Met Care 2008;11:303-308.
5 Gogas H, Trakatelli M, Dessypris N, et al. Melanoma risk in association with serum leptin levels and lifestyle parameters: a case-control study. Annals Oncol 2008;19:384-389.

6 Torisu-Itakura H, Lee JH, Scheri RP, et al. Molecular characterization of inflammatory genes in sentinel and nonsentinel nodes in melanoma. Clin Cancer Res 2007;13:3125-3132.

7 Brandon EL, Cantwell L, Gu J-W, et al. Obesity enhances melanoma tumor growth independently of circulating leptin. FASEB J 2007;21:763.3. 